\title{
Evolution of the Correlation between Orthogonal Polarization Patterns in Broad-Area Lasers
}

\author{
Eduardo Cabrera, ${ }^{*}$ Sonia Melle, Oscar G. Calderón, ${ }^{\dagger}$ and J. M. Guerra \\ Departamento de Optica, Universidad Complutense de Madrid, Ciudad Universitaria s/n, 28040 Madrid, Spain
}

(Received 17 February 2006; published 6 December 2006)

\begin{abstract}
We measure polarization-resolved instantaneous patterns in a large-aspect ratio quasi-isotropic Nd:YAG laser. High correlation between the instantaneous orthogonal polarization patterns recorded at the earlier stages of the laser pulse has been found due to the strong cross saturation between both polarization modes.
\end{abstract}

PACS numbers: 42.65.Sf, 42.60.Jf, 42.60.Mi, 47.54.- $\mathrm{r}$

The vector character of the electric field in lasers plays an important role in laser dynamics when no selection of the polarization is made as in the case of quasi-isotropic laser systems. The interplay of the polarization degrees of freedom can lead to antiphase oscillations between two orthogonal polarization eigenstates in a great variety of lasers, such as fiber lasers [1,2], Nd:YAG lasers [3,4], VCSELs [5], $\mathrm{CO}_{2}$ lasers [6], and Nd-doped microchip glass lasers [7]. Examples of applications based on polarization dynamics are transmission of encoded information $[8,9]$, heterodyne detection systems [10], Doppler velocimetry [11], and optical microwave systems [12].

Novel states of laser light were predicted by San Miguel due to the combination of the polarization state and the transverse spatial profile of the laser field [13]. He studied the spatial polarization instabilities close to laser threshold by means of the vector complex Ginzburg-Landau equation for the physical model corresponding to atomic transitions between spin sublevels $J=1$ and $J=0$. This type of model has been used by Leyva et al. to reproduce successfully the light-polarization dynamics of $\mathrm{CO}_{2}$ lasers [6], also studied by Taggiasco et al. [14]. They observed a doughnutlike intensity profile which consists of a TEM 10 mode polarized along one eigendirection and a $\mathrm{TEM}_{01}$ mode polarized along the orthogonal eigendirection. This phenomenon is found for a cavity detuning close to the gain peak, whereas a cavity detuning far from resonance leads to a doughnutlike mode polarized along one of the eigendirections.

Transverse effects and antiphase dynamics have been also studied in multilongitudinal-mode solid-state lasers and anisotropic lasers by Otsuka [15]. He found that the polarization mode with smaller cross section grows with a high transverse mode whereas the orthogonal polarization mode develops the fundamental mode. In recent years, researchers have focused their attention mainly on vertical cavity surface emitting lasers (VCSEL's) due to the huge Fresnel number that can be obtained in this type of systems and their applications in optical communications. The basic modeling of semiconductor lasers dynamics taking into account the polarization of the laser field was carried out in a seminal work by San Miguel et al. [16]. They used a four-level model corresponding to the spin sublevels of the conduction and valence bands of a semiconductor. Ackemann et al. experimentally found in a VCSEL that one polarization is rather dominant, though the orthogonal one is never completely off [17]. They also observed flips between polarization states. Fratta et al. showed different spatial intensity profiles for the dominant and the weaker polarization modes [18]. While the dominant component has a Gaussian-like pattern, the weaker component developed a four-lobed profile. This difference in the spatiotemporal dynamics for different polarizations has also been seen by Hegarty et al. in a VCSEL with a very large transverse section and short cavity length [19]. They found a polarization instability which leads to a pattern formed by two perpendicular traveling waves with orthogonal polarizations. Recently, Chen et al. have seen vector polarization stationary patterns with different spatial structures for both orthogonal polarization modes [20]. These modes are phase synchronized to a common frequency which is the essential condition needed for a vector polarization pattern. They also found vector vortices lattices and reproduce the spatial profile by using the $\mathrm{SU}(2)$ coherent states (see Ref. [21] for a detailed explanation of the use of these functions).

All of these features point out the relevance of the polarization dynamics in the pattern formation. Previous works have focused on average measurements of transverse patterns or on stationary patterns of $\mathrm{cw}$ lasers. However, these average patterns can mask a rich scenario of transverse dynamics [22]. To the best of our knowledge, the behavior of the interplay between orthogonal polarization patterns along the evolution of the output emission has not been measured. In order to address this question polarization-resolved instantaneous patterns should be recorded which is a great challenge due to the fast evolution of the local intensity. In this Letter we present polarizationresolved instantaneous snapshots of the pattern dynamics in a broad-area flash-lamp pumped Nd:YAG laser. This laser shows antiphase oscillations in the temporal evolution of two orthogonal polarization components, as it has been recently reported in Ref. [4]. Therefore a high degree of correlation may be expected between the spatial profiles of these modes. Our measurements will allow following this correlation along the evolution of the output pulse and 
determining the fundamental parameters on which this correlation depends.

The laser is an air-cooled, low pulse energy $(\simeq 50 \mathrm{~mJ})$ flash-lamp pumped prototype (see Ref. [4] for a detailed description). The lasing medium was a Nd:YAG cylindrical rod ( $60 \mathrm{~mm}$ long $\times 6 \mathrm{~mm}$ diam), side pumped by two parallel linear flash lamps. In this form, the pumping geometry and polarizations break the symmetry between the horizontal and vertical axis inducing the polarization antiphase behavior and selecting a "Cartesian symmetry" instead of a circular symmetry. It works in a single-shot regime with a pulse length of $100 \mu \mathrm{s}$, leaving enough time between shots to avoid thermal effects. The Fresnel number is close to a value of 50, which allows the system to excite a high number of modes simultaneously, and thus, to develop very complex patterns. The pumping is fixed to 2.1 times above threshold. A polarizing beam splitter was placed behind the output coupler to separate the beam in horizontal $(H$ mode) and vertical $(V$ mode) polarization components. Each beam is split again by a nonpolarizing beam splitter (50:50) in order to register the temporal evolution of each component with two photodiodes (1 ns rise time). After those steps both polarization patterns simultaneously reach, in separated areas, the microchannel plate of an intensified IR enhanced CCD camera with a $768 \times 494$ pixels array. The spatial resolution is $50 \mu \mathrm{m} /$ pixel which allows one to observe the spatial structures forming the patterns. The minimum integration time for the recorded patterns is $1 \mathrm{~ns}$, much lower than the characteristic time of the local intensity evolution. We take one snapshot per pulse. Both the integration time and the time within the pulse at which we record the snapshots (time delay) are computer controlled. Because of the jitter inherent to the measure system this time delay has an uncertainty of few $\mu$ s.

Figure 1 shows four pairs of different instantaneous patterns corresponding to both polarization modes for different times along the pulse. The spatial intensity profile of each polarization mode crosses through different stages from boundary-controlled patterns to turbulentlike patterns. Similar behavior has been recently reported in this laser for the intensity pattern evolution without resolving polarization [23]. We can see in Fig. 1(a) that both components develop the same spatial profile in the first steps of the evolution. This similarity reveals a strong coupling between both modes. However, this coupling is not strong enough to keep a high correlation for longer times, when the nonlinearities start to play an important role. Figure 1(b) shows an intermediate time before the patterns reach the disordered stage. Both polarization modes present a high degree of order, but with very different spatial profiles. In these steps the system presents a great variety of spatial profiles: latticelike patterns, stripelike modes, and flowerlike modes. For longer times these patterns are destabilized in a different way for each polarization mode toward completely disordered patterns with no relation between both modes [see Fig. 1(d)]. The time at

which the transition to disorder takes place is almost, but not exactly, the same for the $V$ mode and for the $H$ mode. This feature is shown in Fig. 1(c) where the $V$ mode (on the left) develops a disordered pattern, while the pattern corresponding to the $H$ mode (on the right) is still formed by a superposition of stationary waves, presenting some degree of order. Unfortunately, the lack of repetition between different pulses does not allow us to measure precisely the transition time for both modes.

In order to quantify the evolution of the degree of similarity between the orthogonal polarization patterns

(a)

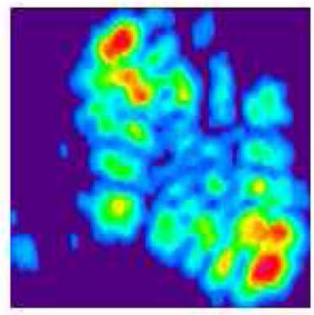

(b)

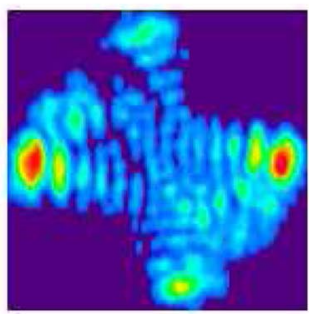

(c)

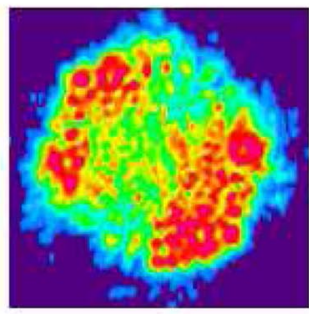

(d)
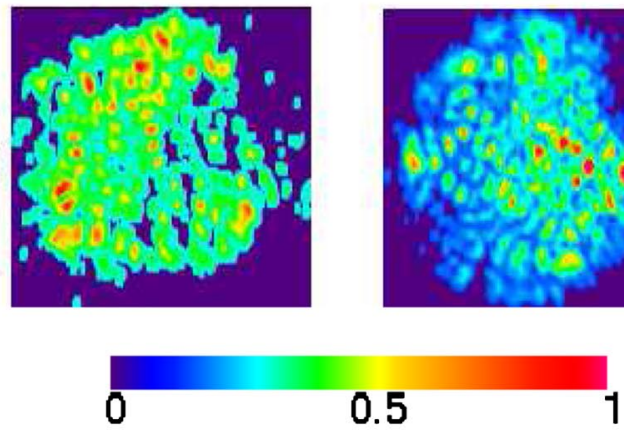

FIG. 1 (color online). Instantaneous intensity patterns (resolution time $15 \mathrm{~ns}$ ) for a pumping 2.1 times above threshold. The left column corresponds to the $V$ mode, while the right column corresponds to the $H$ mode: (a) for $11 \mu$ s of time delay from the beginning of the pulse; (b) for $17 \mu$ s of delay; (c) for $32 \mu \mathrm{s}$ of delay; and (d) for $63 \mu \mathrm{s}$ of delay. Pattern size $6 \times 6 \mathrm{~mm}$ approximately. 
( $V$ mode and $H$ mode), we calculate their cross correlation at different times along the output pulse. Before computing the correlation we have removed from the images their slow variation profile. This process allows one to extract the spatial structures from the whole pattern. We plot in Fig. 2 the cross correlation between both polarization patterns versus the time delay along the pulse. Each point corresponds to the mean correlation for experiments recorded at the same time delay fixed by the camera controller from the beginning of the pulse. The jitter inherent to the measure system can be seen in the horizontal error bars. Thus, each point represents not only one specific case, but a tendency of the system repeated in every output pulse evolution. It can be seen that at the beginning of the emission a high correlation close to 1 takes place, which points out that the patterns for both polarization eigenstates show the same features. However, as time increases, the correlation decreases quickly until it reaches a plateau value close to 0.2 , for which the patterns are quite dissimilar. For the pumping used in the experiments, the time needed to decorrelate both patterns is approximately $15 \mu \mathrm{s}$, smaller than the transition time toward disorder (which is around $30 \mu \mathrm{s}$ ).

The theoretical model used to discuss the experimental results is based on the existence of two laser subsystems associated with two orthogonal polarization eigenstates. Bielawski et al. [1], Lacot et al. [2], and Cabrera et al. [4] have used this model to explain the antiphase polarization dynamics in fiber lasers [1,2] and Nd:YAG lasers [4], showing a good agreement with the experiments. We extend this model to include the spatial degrees of freedom. Thus, laser equations can be written in the following form:

$$
\frac{d E_{V}}{d t}=\kappa\left[D_{V}+\beta D_{H}-\eta+i a \nabla_{\perp}^{2}\right] E_{V}
$$

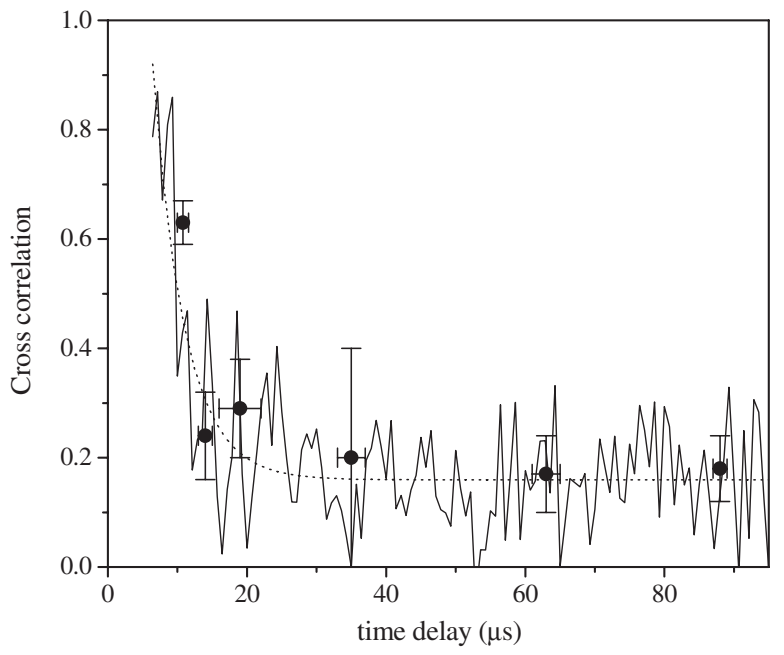

FIG. 2. Evolution of the correlation between both polarization patterns.: (dots) experimental points averaged for experiments recorded with the same time delay from the beginning of the pulse; (solid line) correlation between simulated patterns; (dashed line) exponential fit to data.

$$
\begin{aligned}
\frac{d E_{H}}{d t} & =\kappa\left[D_{H}+\beta D_{V}-\eta+i a \nabla_{\perp}^{2}\right] E_{H}, \\
\frac{d D_{V}}{d t} & =\gamma_{\|}\left[r-\left(1+\left|E_{V}\right|^{2}+\beta\left|E_{H}\right|^{2}\right) D_{V}\right], \\
\frac{d D_{H}}{d t} & =\gamma_{\|}\left[r-\left(1+\left|E_{H}\right|^{2}+\beta\left|E_{V}\right|^{2}\right) D_{H}\right],
\end{aligned}
$$

where $E_{V}$ and $D_{V}\left(E_{H}\right.$ and $\left.D_{H}\right)$ are the dimensionless slowly varying amplitude of the electric field and of the population inversion, respectively, associated to the vertical (horizontal) polarization eigenstate. $1 / \kappa \simeq 7 \mathrm{~ns}$ is the time decay of the electric field (calculated taking into account the mirror losses), and $1 / \gamma_{\|} \simeq 0.23$ is the population inversion lifetime. $r$ represents the dimensionless pumping parameter. In order to reproduce the experimental conditions, we use a square spatial pumping profile. Likewise, the temporal form of the pumping was simulated by a function approximating the pulse excitation of the lamps. $\eta(x, y)$ takes into account the spatial dependence of the losses. Light diffraction is taken into account by means of the transverse Laplacian term in field equations, and is measured by the diffraction coefficient $a=c \lambda /\left(4 \pi \kappa b^{2}\right)$, where $b=6 \mathrm{~mm}$ is the spatial transverse size of the laser and $\lambda=1.06 \mu \mathrm{m}$ is the laser wavelength. $\nabla_{\perp}^{2}=\partial_{x}^{2}+\partial_{y}^{2}$ is the transverse Laplacian where $x$ and $y$ are normalized with the spatial scale $b$. $\beta$ is the cross-saturation coefficient describing how each laser field is coupled with the population inversion of the other laser subsystem. The value used for this coefficient is obtained experimentally from the frequency of the relaxations oscillations $f_{R}$ and the frequency of the antiphase oscillations between both orthogonal polarizations $f_{L}$ as $\beta=\left(f_{R}-f_{L}\right) /\left(f_{R}+f_{L}\right) \simeq$ 0.8 (see Ref. [4] for details).

Integration of the system of Eqs. (1) and (4) gives qualitative and quantitative agreement with experimental results. We plot in Fig. 3 four representative pairs of simulated patterns corresponding to both polarization modes showing the same behavior found in the experiments. In the first steps of the output emission, both polarization modes develop similar spatial profiles [see Fig. 3(a)]. At intermediate times, both patterns are still highly ordered but they present different spatial profiles [see Fig. 3(b)]. After that, both modes continue changing to reach a disorder stage, although they do it at different times [see Fig. 3(c)]. Finally, both polarization modes have a completely irregular spatial shape [see Fig. 3(d)].

Once the model has reproduced the main features of the dynamics, let us study the role of the parameter $\beta$ that controls the coupling strength between both orthogonal polarization subsystems. Simulations made with different values of $\beta$ show that the cross-saturation process is the main factor that determines the resemblance between both polarization patterns, as it was expected. Low values of $\beta$, such as 0.2 , cause both modes to grow with different spatial profiles since the early stages of the evolution, while as $\beta$ gets closer to 1 the time the system needs to decorrelate 
(a)
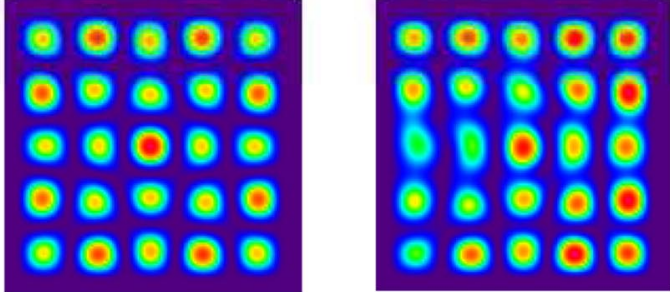

(b)
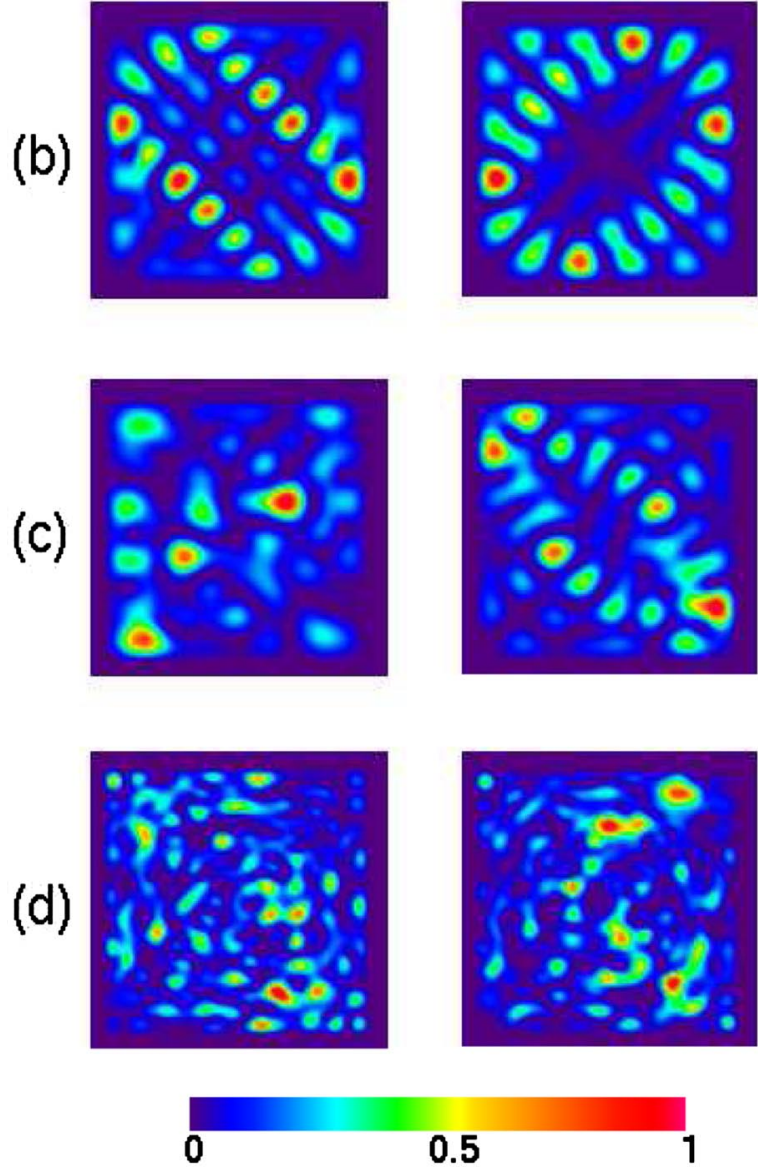

\section{5}

FIG. 3 (color online). Simulated intensity patterns for $H$ mode (left column) and for $V$ mode (right column) corresponding to different delays along the laser pulse: (a) $10 \mu \mathrm{s}$, (b) $12 \mu \mathrm{s}$, (c) $15 \mu \mathrm{s}$, and in the middle of the pulse (d) $42 \mu \mathrm{s}$. Patterns obtained for the pumping value used in the experiment.

both modes increases. The reason for that behavior lies in the spatial distribution $f(\theta)$ of the induced electric dipoles, which determines the cross-saturation strength. Completely uncorrelated dipoles, i.e., dipoles oriented in orthogonal directions, give a zero value of $\beta$. On the other hand, as $f(\theta)$ becomes narrower around $\theta=45^{\circ}, \beta$ approximates to 1 , which means completely correlated polarizations.

In conclusion, instantaneous snapshots have been recorded to follow the spatiotemporal dynamics of the vector polarization pattern in a broad-area Nd:YAG laser. As it was expected a high degree of spatial correlation has been found between both polarization orthogonal modes. Despite the temporal antiphase dynamics last the whole pulse, the system cannot maintain the similarity between the spatial profiles. Furthermore, the loss of correlation along the pulse occurs at times smaller than the time needed for the pattern to become disordered. The simulations reveal that the cross-saturation mechanism is responsible for the high correlation found during the first stages of pulse emission.

We wish to acknowledge Paul Hoess for technical support. This work was supported by Project No. BFM200306292 (Spain).

*Electronic address: ecabrera@ fis.ucm.es

${ }^{\dagger}$ URL: http://www.ucm.es/info/laserlab

[1] S. Bielawski, D. Derozier, and P. Glorieux, Phys. Rev. A 46, 2811 (1992).

[2] E. Lacot, F. Stoeckel, and M. Chenevier, Phys. Rev. A 49, 3997 (1994).

[3] P. Khandokhin et al., in Laser Optics'98: Solid State Lasers, edited by V.I. Ustyugov, Proc. SPIE Int. Soc. Opt. Eng. Vol. 3682 (SPIE-International Society for Optical Engineering, Bellingham, WA, 1998), pp. 53-62.

[4] E. Cabrera, O. G. Calderón, and J. M. Guerra, Phys. Rev. A 72, 043824 (2005).

[5] F. Prati, G. Giacomelli, and F. Marin, Phys. Rev. A 62, 033810 (2000).

[6] I. Leyva, E. Allaria, and R. Meucci, Phys. Rev. A 68, 053806 (2003).

[7] B. Peters et al., Phys. Rev. A 64, 023816 (2001).

[8] E. A. Viktorov and P. Mandel, Opt. Lett. 22, 1568 (1997).

[9] G.D. VanWiggeren and R. Roy, Phys. Rev. Lett. 88, 097903 (2002).

[10] G. W. Baxter et al., IEEE Photonics Technol. Lett. 8, 1015 (1996).

[11] P. Nerin et al., Electron. Lett. 33, 491 (1997).

[12] M. Brunel, F. Bretenaker, and A. Le Floch, Opt. Lett. 22, 384 (1997).

[13] M. San Miguel, Phys. Rev. Lett. 75, 425 (1995).

[14] C. Taggiasco et al., Opt. Commun. 133, 507 (1997).

[15] K. Otsuka, IEEE J. Quantum Electron. 14, 49 (1978); Jpn. J. Appl. Phys. 32, L1414 (1993); Chaos Solitons Fractals 4, 1547 (1994).

[16] M. San Miguel, Q. Feng, and J. V. Moloney, Phys. Rev. A 52, 1728 (1995).

[17] T. Ackemann et al., J. Opt. B 2, 406 (2000).

[18] L. Fratta et al., Phys. Rev. A 64, 031803(R) (2001).

[19] S. P. Hegarty et al., Phys. Rev. Lett. 82, 1434 (1999).

[20] Y.F. Chen et al., Phys. Rev. Lett. 90, 053904 (2003).

[21] Y. F. Chen, K. F. Huang, and Y. P. Lan, Phys. Rev. E 66, 046215 (2002).

[22] F. Encinas-Sanz, S. Melle, and O. G. Calderón, Phys. Rev. Lett. 93, 213904 (2004).

[23] E. Cabrera et al., Opt. Lett. 31, 1067 (2006). 